\title{
Model Order Reduction for Reliability Assessment of Flexible Power Networks
}

\author{
Mike Brian Ndawula,*, Ignacio Hernando-Gil ${ }^{\mathrm{b}}$, Ran $\mathrm{Li}^{\mathrm{a}}$, Chenghong $\mathrm{Gu}^{\mathrm{a}}$, \\ Antonio De Paola ${ }^{a}$ \\ ${ }^{a}$ Centre for Sustainable Power Distribution, University of Bath, Bath BA2 7AY, UK \\ ${ }^{b}$ ESTIA Institute of Technology, University of Bordeaux, F-64210 Bidart, France
}

\begin{abstract}
Model order reduction (MOR) has demonstrated its robustness and wide applicability in simulating large-scale mathematical models in the engineering research domain. In this paper, MOR techniques are applied to quantify relevant reliability metrics of power distribution systems and the impact associated with the integration of different smart grid technologies. To the best of the authors' knowledge, this is the first application of MOR techniques of balanced truncation to derive reliability models of electricity networks, which exhibit a reduced number of equivalent components and thus simplify the complexity for network analysis. The extensive case studies presented, based on both radial and meshed systems, demonstrate that the proposed technique allows for a faster reliability assessment through Monte Carlo simulation while preserving high accuracy. The proposed methodology can also be applied to systems endowed with photovoltaic and energy storage technologies, emphasising that this approach represents a promising starting point for reliability analysis of more complex systems, which are normally characterised by a large penetration of these distributed energy resources.
\end{abstract}

Keywords: balanced truncation, distributed energy resources, distribution networks, model order reduction, Monte Carlo simulation, system reliability

\footnotetext{
*Corresponding author

Email addresses: m.b.ndawula@bath.ac.uk (Mike Brian Ndawula), i.hernandogil@estia.fr (Ignacio Hernando-Gil), R.Li2@bath.ac.uk (Ran Li), C.Gu@bath.ac.uk (Chenghong Gu), A.De.Paola@bath.ac.uk (Antonio De Paola)
} 


\begin{tabular}{ll}
\hline Acronyms & \\
\hline AEM & Alternative Existing Method \\
BT & Balanced Truncation \\
CAIDI & Customer Average Interruption Duration Index \\
CAIFI & Customer Average Interruption Frequency Index \\
CoV & Coefficient of Variation \\
CTMC & Continuous Time Markov Chain \\
DNO & Distribution Network Operator \\
ENS & Energy Not Supplied \\
ES & Energy Storage \\
HPC & High Performance Computing \\
HSV & Hankel Singular Value \\
LP & Load Point \\
MCS & Monte Carlo Simulation \\
MESS & Matrix Equation Sparse Solver \\
MOR & Model Order Reduction \\
OR & Order Reduction \\
PC & Power Component \\
PNS & Power Not Supplied \\
PSS ${ }^{\circledR}$ & Power System Simulator for Engineering \\
PV & Photovoltaic \\
RBTS & Roy Billinton Test System \\
SAIDI & System Average Interruption Duration Index \\
SAIFI & System Average Interruption Frequency Index \\
TNR & Traditional Network Reduction \\
&
\end{tabular}

\section{Nomenclature}

$\hat{H}(s)$ transfer function of the reduced system

$\hat{y}(\cdot) \quad$ output of the reduced system

$\lambda$ component failure rate

$\|\cdot\|_{\infty} H_{\infty}$-norm of a rational transfer function 
$\mu \quad$ component repair rate

$\sigma \quad \mathrm{HSV}$ of a system

A system matrix or state transition matrix

$B \quad$ control/input matrix

C output matrix

$H(s)$ transfer function of the original system

$N \quad$ number of samples

$n \quad$ state space dimension of the original system

$P \quad$ controllability Gramian

Q observability Gramian

$r \quad$ state space dimension of the reduced system

$T \quad$ transformation matrix

$U$ unavailability

$u(\cdot) \quad$ input

$x(t)$ state vector

$y(\cdot) \quad$ output of the original system

\section{Introduction}

Power systems are inherently complex and can often be accurately described only by using models with several variables, depending on the intended application. Since complex models are computationally expensive to simulate, it is common practice to use simplified representations of the power system for analysis and design purposes. Reduced models might also be necessary due to other practical reasons, for example when only a limited number of measurements are available for system monitoring, or in the case of interconnected power systems whose single areas (owned by different utilities) are reluctant to share complete and detailed system information [1]. Even with systematic problem decomposition, the pragmatic choice is often to use reduced versions of the original network to run system simulations, where the 
computational complexity depends at least polynomially on the size of the network [2].

The employment of simplified network models has always been very common in reliability analyses of distribution networks [3, 4] and it is becoming even more relevant as the level of complexity in power systems rapidly increases [5], following the integration of photovoltaic renewable generation (PV) and technologies such as energy storage (ES), electric vehicles and demand response actuators. In a context of growing complexity, it will be necessary to use simplified benchmark models to accurately assess network reliability and determine efficient future investments for a flexible and secure power grid with appropriate reliability standards [6].

This paper proposes the utilisation of model order reduction (MOR) tools to develop accurate reduced models of distribution networks for reliability purposes. A rigorous analytical method is used to obtain simplified grid representations that minimise the estimation error of relevant reliability indexes while ensuring significantly shorter computational times. Furthermore, the proposed methodology can easily accommodate the inclusion of PV and ES technologies and quantify their impact in terms of reliability. The rest of the paper is organised as follows: the state of the art is reviewed in the next section, and the background theory of MOR is explored in Section 3. Section 4 details the development of the methodology using MOR for reliability assessment. Section 5 evaluates the performance of the proposed approach on different case studies and conclusive remarks are presented in Section 6.

\section{Network Reduction in Power System Analysis - Related Works}

It is common in network studies to simplify the utilised models to obtain a system description with the best trade-off between accuracy and complexity. In these cases, the size of the network models is generally reduced by substituting sets of connected elements (buses, lines, transformers, etc.) and transforming them into smaller and numerically equivalent systems [7]. Typical applications of this approach include symmetrical or asymmetrical short circuit calculations and load flow calculations. In these cases, the performance of the reduced networks representations is evaluated in terms of accuracy of the power flow results with respect to the (more complex) initial model [8].

The typical approach for network reduction in a reliability context is to simplify the system representation by systematically replacing certain connected 
elements of the chosen reliability model (e.g., series and parallel configurations in reliability block diagrams) with fewer equivalent components exhibiting the same reliability properties. The main drawbacks of this method of network reduction are a) its limited applicability, i.e. only to networks with relatively simple topology [9]; b) it cannot be used to calculate customerbased reliability indices such as customer average interruption duration and frequency indices (CAIDI and CAIFI) [10] because it does not allow for an accurate aggregation of demand at different network nodes; c) the impact of critical or unreliable areas and components on the system reliability metrics becomes increasingly harder to distinguish; d) it is difficult to accommodate some relevant reliability features such as different modes of failure, maintenance and weather effects. Despite those drawbacks, this method is useful in practice, particularly for simple analyses where analytical refinements are not desired [4]. Given these limitations, alternative approaches have been developed, such as the decomposition method, which is based on conditioning a complex system on the state of a key power component (PC) [9]. However, this method is not suitable for large systems because, as the number of key PCs increase, the model quickly becomes unmanageable. There also exist analysis algorithms based on testing minimal paths (or using the minimal cut set approach) [9] but their main drawback is that, for large systems, the increased number of paths and cut sets leads to a combinatorial explosion.

This research explores a new approach to network reduction for reliability assessment purposes based on MOR. The chosen method relies on singular value decomposition and balanced truncation. The dynamical system describing the reliability of the network is simplified by first calculating its Hankel singular values (HSVs) [11], which indicate the relevance of each system state in terms of reliability, and then the dynamics which have a smaller impact on the considered reliability indexes are neglected. Model Reduction has already been applied in various power system analyses: MOR using balanced empirical Gramians was investigated for linear systems in [12, 13, 14] and nonlinear systems in $[15,16,17,18,19]$. Given the need to reduce large power systems, [20] used a linear system reduction method and the work was successful for small-signal stability while [21] performed a parametric MOR aimed at preserving parameters related to decentralised power system devices such as stabilisers. Additionally, MOR has been used to obtain reduced models of PV systems [22, 23], battery energy storage systems [24] and of microgrids [25], using the singular perturbation technique. Albeit not 
explored in this paper, another important class of MOR methods is based on Krylov subspaces (moment matching). It was used for reduction of a large-scale multiport piezo energy harvester in [26] while in [27] and [28], the technique was extended to reduce large scale power systems and interconnected systems, respectively.

\subsection{Article Contributions}

This work proposes a novel approach for the creation of simplified network models for reliability assessment purposes. To the best of our knowledge, this is the first attempt to apply model order reduction (MOR) in this context and analytically derive simplified grid representations that contain the most important system dynamics and, at the same time, minimise the error of the considered grid reliability metrics. Time-sequential Monte Carlo simulations (MCSs) are carried out on systems of varying complexity to verify that the resulting reduced models provide a reasonably accurate reliability assessment while being faster to simulate. It is also shown that the proposed technique is able to effectively quantify the impact of PV and ES on network reliability. The proposed methodology is presented in Fig. 1.

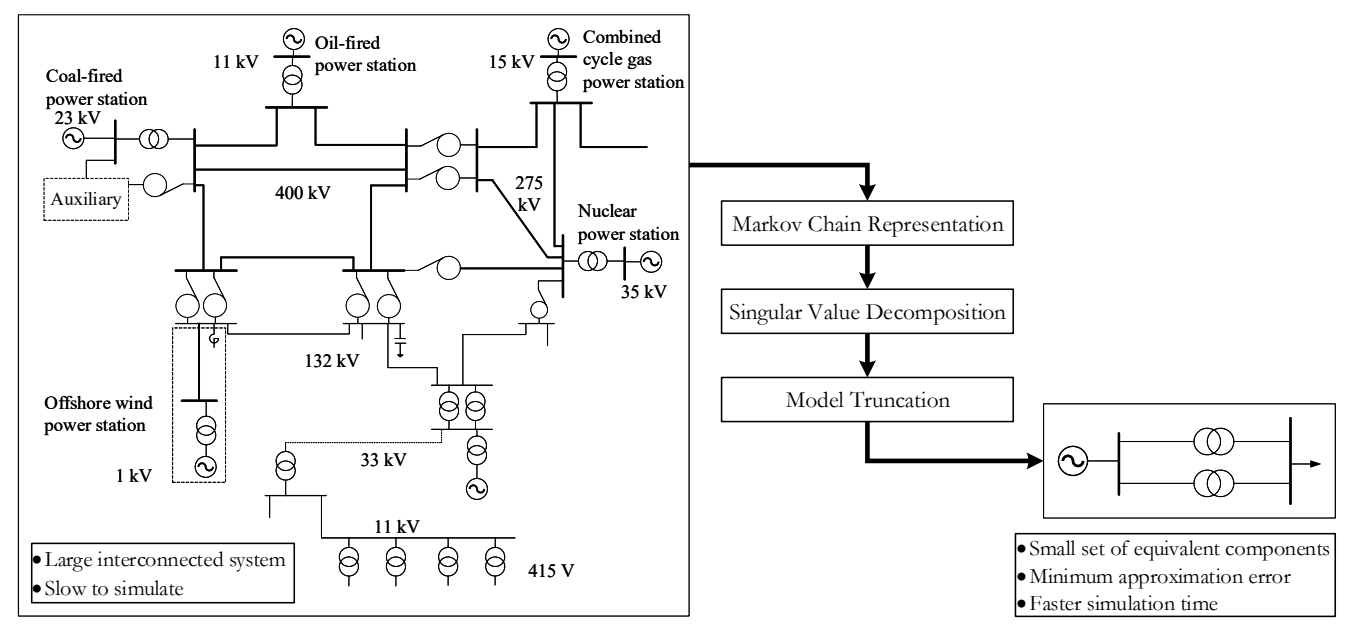

Figure 1: Representation of the proposed methodology.

\section{MOR Theory}

This section details the general theoretical framework of MOR and balanced truncation (BT) while the application of this technique to the specific prob- 
lem of deriving simplified reliability models for distribution networks is presented in Section 4.

Let us consider the following linear time-invariant system with input $u$ and output $y$ :

$$
\begin{aligned}
\dot{x}(t) & =A x(t)+B u(t), \\
y(t) & =C x(t),
\end{aligned}
$$

The internal dynamics of the system are represented by the state vector $x(t) \in \mathbb{R}^{n}$, whose evolution over time is determined by the system matrix $A \in$ $\mathbb{R}^{n x n}$ and by the input matrix $B \in \mathbb{R}^{n x m}$. In the present formulation, there is no direct input/output relationship and the system output $y(t)$ corresponds to a linear combination of the state $x(t)$, according to the output matrix $C \in \mathbb{R}^{q x n}$.

Model order reduction (MOR) aims at reducing the order $\mathrm{n}$ of the system while preserving the fundamental relationships between its inputs and outputs. To this end, the state vector $x(t)$ is projected onto a low-dimensional subspace, neglecting the less relevant system dynamics. The MOR is performed to minimise, for any input $u(\cdot)$, the error between the output response of the reduced model $\hat{y}(\cdot)$ and the one of the original model $y(\cdot)$ [29].

We restrict our attention to stable systems, i.e. system which has a matrix $A$ with all its eigenvalues in the open left half of the complex plane $\mathbb{C}^{-}$. We are interested in constructing a reduced-order system as in (2):

$$
\begin{aligned}
\dot{\hat{x}}(t) & =\hat{A} \hat{x}(t)+\hat{B} u(t), \\
\hat{y}(t) & =\hat{C} \hat{x}(t),
\end{aligned}
$$

where $\hat{A} \in \mathbb{R}^{r x r}, \hat{B} \in \mathbb{R}^{r x m}, \hat{C} \in \mathbb{R}^{q x r}$, and $r<n$. In the present context of linear systems, BT is one of the preferred methods for model order reduction since it preserves stability and provides a global computable error bound between the transfer functions of the original and the reduced-order system. This notion is expressed through the transfer functions $H(s), \hat{H}(s) \in \mathbb{C}^{q x m}$ of the original and reduced system, respectively, which denote the relationships between the input signal and the resulting output response in the frequency domain. An expression for the error between the original and the reduced 
system output, given by (3), is obtained by driving both systems with the same input $u(\cdot)$ :

$$
\|y-\hat{y}\|_{2} \leq\|H-\hat{H}\|_{\infty}\|u\|_{2}
$$

where $\|\cdot\|_{2}$ is the Euclidean norm and $\|\cdot\|_{\infty}$ is the $H_{\infty}$-norm of a rational transfer function [29].

\subsection{Balanced Truncation}

The fundamental principle of BT for MOR relies on the notions of controllability and observability matrices $P$ and $Q$, as defined in (4) [30]:

$$
P=\int_{0}^{\infty} e^{A t} B B^{t} e^{A^{T} t} d t, \quad Q=\int_{0}^{\infty} e^{A^{T} t} C^{T} C e^{A t} d t
$$

In broad terms, the controllability Gramian $P$ determines how much the inputs $u$ affect each component in the state $x$. Similarly, the observability Gramian $Q$ quantifies the impact of each state component in $x$ on the system outputs $y$. The first step of the BT technique is a change of coordinates in the original system (1), according to the transformation matrix $T \in \mathbb{R}^{n x n}$ :

$$
\begin{aligned}
& \dot{\tilde{x}}(t)=T A T^{-1} \tilde{x}(t)+T B u(t), \\
& y(t)=C T^{-1} \tilde{x}(t),
\end{aligned}
$$

It is possible to demonstrate that the matrix $T$ can always be chosen to obtain new Gramians $\tilde{P}$ and $\tilde{Q}$ which are equal and diagonal:

$$
\tilde{P}=T P T^{T}=\tilde{Q}=T^{-T} Q T^{-1}=\sum=\operatorname{diag}\left(\sigma_{1}, \sigma_{2}, \ldots, \sigma_{n}\right),
$$

In equation (6), the terms $\sigma_{1}, \ldots, \sigma_{n}$ denote (in non-increasing order) the Hankel Singular Values (HSVs) of system (1), which broadly speaking provide a measure of energy for each system state. The state vector $\tilde{x}$ in (5) can now be partitioned as $\tilde{x}(t)=\left[\begin{array}{l}\tilde{x}_{1}(t) \\ \tilde{x}_{2}(t)\end{array}\right]$, where $\tilde{x}_{1}(t) \in \mathbb{R}^{r}$ contains the $r$ state components associated to the highest HSVs of the system and indicates the dimension of the reduced system. The equations in (5) can be rewritten as: 


$$
\begin{aligned}
& \tilde{x}(t)=\left[\begin{array}{ll}
\tilde{A}_{11} & \tilde{A}_{12} \\
\tilde{A}_{21} & \tilde{A}_{22}
\end{array}\right]\left[\begin{array}{l}
\tilde{x}_{1}(t) \\
\tilde{x}_{2}(t)
\end{array}\right]+\left[\begin{array}{c}
\tilde{B}_{1} \\
\tilde{B}_{2}
\end{array}\right] u(t), \\
& \tilde{y}(t)=\left[\begin{array}{ll}
\tilde{C}_{1} & \tilde{C}_{2}
\end{array}\right]\left[\begin{array}{l}
\tilde{x}_{1}(t) \\
\tilde{x}_{2}(t)
\end{array}\right],
\end{aligned}
$$

The reduced-order system (2) can then be obtained by simple truncation $[29,31]$ from the balanced realisation (7) in partitioned form, with:

$$
\hat{A}=\tilde{A}_{11} \in \mathbb{R}^{r x r}, \quad \hat{B}=\tilde{B}_{1} \in \mathbb{R}^{r x m}, \quad \hat{C}=\tilde{C}_{1} \in \mathbb{R}^{q x r},
$$

A key fundamental result of $\mathrm{BT}$ is that the global error between $H(\cdot)$ and $\hat{H}(\cdot)$, i.e. between the transfer functions of the original and reduced system, fulfils the following condition:

$$
\|H-\hat{H}\|_{\infty} \leq 2\left(\sigma_{r+1}+\sigma_{r+2}+\ldots+\sigma_{n}\right)
$$

where $\sigma_{r+1}, \ldots, \sigma_{n}$ are the neglected HSVs. This means that the dimension $r$ of the reduced system can be selected to achieve the desired trade-off between system size and model accuracy.

\subsection{Model Order Reduction of Systems without Inputs}

As shown later on, the dynamical system considered in this work for grid reliability assessment does not have any input. This means that some minimal adjustments are required in the MOR technique presented in the previous subsection. In particular, the controllability Gramian $P$ is not well defined and the matrix $T$ for the change of coordinates in the original system is calculated to only diagonalize the observability Gramian $Q$ :

$$
\tilde{Q}=T^{-1} Q T=\sum=\operatorname{diag}\left(\sigma_{1}, \sigma_{2}, \ldots, \sigma_{n}\right),
$$

The reduced-order system is then determined according to (5)-(8). In this specific case, the error bound of the model order reduction can be derived on the quadratic norm of the output. Recalling that the natural response of a linear system with initial state $x_{0}$ is equal to $y=C e^{A t} x_{0}$, in the changed coordinates we have: 


$$
\begin{aligned}
\int_{0}^{\infty}\|\tilde{y}(t)\|_{2}^{2} d t=\int_{0}^{\infty} \tilde{x}_{0}^{T} e^{\tilde{A}^{T} t} \tilde{C}^{T} \tilde{C} e^{\tilde{A} t} \tilde{x}_{0} d t \\
\\
=\tilde{x}_{0}^{T} \tilde{Q} \tilde{x}_{0}=\tilde{x}_{0}^{T} \cdot \operatorname{diag}\left(\sigma_{1}, \sigma_{2}, \ldots, \sigma_{n}\right) \cdot \tilde{x}_{0} .
\end{aligned}
$$

It follows that, if the reduced-order model is obtained according to the truncation presented in (8), we have the following error bound on the outputs $\tilde{y}$ and $\hat{y}$ of the original and reduced-order system:

$$
\int_{0}^{\infty}\|\tilde{y}(t)\|_{2}^{2}-\|\hat{y}(t)\|_{2}^{2} d t \leq \sum_{i=r+1}^{n} \sigma_{i} \quad \forall \tilde{x}_{0}:\left\|\tilde{x}_{0}\right\|_{1}=1 .
$$

\section{MOR for Reliability Assessment}

The main aim of the proposed methodology is to develop a tool capable of creating simplified grid models that preserve the key features of the original network and allow for accurate and faster reliability analyses. This section presents the methodology for developing the system model, performing the model order reduction, and finally carrying out time-sequential MCS analyses to quantify the reliability metrics of interest.

\subsection{Modelling System Descriptor Matrices}

This section describes how the MOR techniques discussed in Section 3 can be applied to the specific case of reliability assessment of distribution networks. As a first step, the working state of a generic grid is described as a continuous-time Markov chain (CTMC), under the common assumption of Poisson distributions for the fail/repair times of the system PCs. Each discrete state of the CTMC corresponds to a specific reliability state of the system components and the transitions between these states are associated with the failure or the repairing of a certain component. The discrete states and transitions of the CTMC, for the simple case of a system with two repairable components $[3,32]$, are shown in Fig. 2. In this example, each PC has two modes (UP/DOWN), and the failure and repair rates are denoted by lambda and $m u$, respectively. Since we are considering 2 components, the resulting Markov chain will have $2^{2}$ discrete states. The passage from state 1 (with both components UP) to state 2 (with component 1 DOWN and 
component 2 UP) will occur with rate $\lambda_{1}$, i.e. the failure rate of component 1. Conversely, the passage from state 2 to 1 will occur with rate $\mu_{1}$, i.e. the repair rate of component 1 .

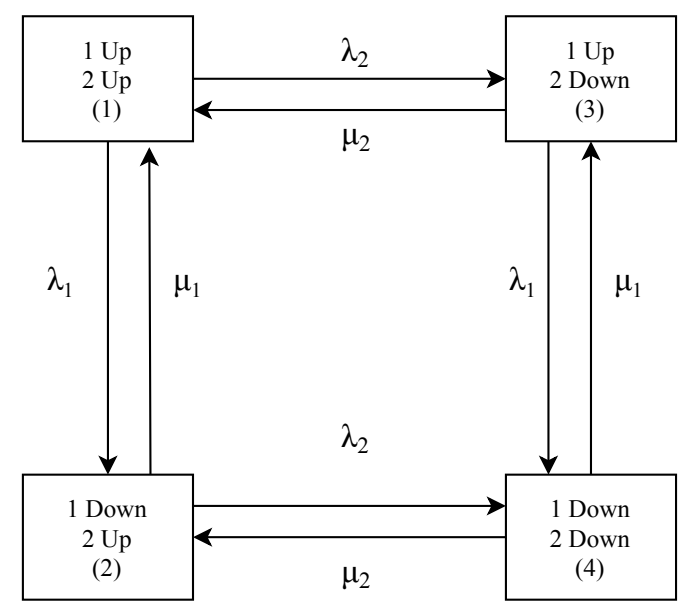

Figure 2: Markov chain representing a system with two repairable components [3].

On this basis, the proposed modelling approach uses a probabilistic description to characterise the state vector in the system (1): the dynamic state $x(t)$ will represent the probability of a specific reliability state of the system PCs (i.e. a specific discrete state of the CTMC) to occur at time t. The evolution of $x(t)$ follows the equations in (1) and the associated state matrix $A$ is given by (13).

$$
A=\left[\begin{array}{cccc}
-\left(\lambda_{1}+\lambda_{2}\right) & \mu_{1} & \mu_{2} & 0 \\
\lambda_{1} & -\left(\lambda_{2}+\mu_{1}\right) & 0 & \mu_{2} \\
\lambda_{2} & 0 & -\left(\lambda_{1}+\mu_{2}\right) & \mu_{1} \\
0 & \lambda_{2} & \lambda_{1} & -\left(\mu_{1}+\mu_{2}\right)
\end{array}\right]
$$

In the present case, the nondiagonal element $a_{i j}$ of $A$ equals to the transition rate from state $i$ to $j$, whereas the diagonal elements $a_{i i}$ are chosen to obtain zero-sum columns since the sums of the rates of all transitions leaving and entering the reliability state must be 0 :

$$
a_{i i}=-\sum_{j \neq i} a_{i j}
$$


Note that the state matrix $A$ corresponds to the transpose of the stochastic transitional probability matrix in [3] and, given its specific properties (zerosum columns and non-negative off-diagonal elements), it is also a Metzler matrix [33].

We wish to emphasise that the proposed modelling approach can also accommodate common mode failures of system components, considering transitions to reliability states that have more than one additional component in DOWN mode. For example, in Fig. 2, a common mode failure of the two system components would be modelled by a transition from state (1) to state (4), specifying its non-negative transition rate as the value of $a_{41}$ in (13). Alternatively, cascaded failures can be modelled as events where the failure of a certain component leads to an increased failure rate of another component. For example, consider the possibility that a failure of PC1 increases the failure rate of $\mathrm{PC} 2$. This would imply that the transition rate from state (2) to state (4) in Fig. 2 (i.e. the element $a_{42}$ in (13)) would be higher than the transition rate $\lambda_{2}$ from state (1) to state (3), i.e. the element $a_{31}$ in (13).

In the present case, we are not considering the terms $B u(t)$ in $(1)$, since the chosen reliability modelling approach does not entail any external input. As previously stated, the network reliability performance is based on uncontrollable fault occurrences and corresponding repairs to faulty PCs that allow for supply restoration and are performed with fixed rates.

The output $y(t)$ of system (1) has dimension 1 and corresponds to the expected value of the Power Not Supplied (PNS) at time $t$. This implies that each term $c_{j}$ in the matrix $\mathrm{C}$ corresponds to the PNS associated to the $\mathrm{k}^{\text {th }}$ reliability state of the system. For example, considering again that $x_{1}$ denotes the probability that all the system PCs are in the UP mode, we will have $c_{1}=0$. With this approach, the Energy Not Supplied (ENS) in the system can be calculated by taking the integral of $y(t)$.

\subsection{MOR Implementation}

This subsection details the actual implementation of the proposed approach for network reliability assessment. The analysis will consider the ENS index as the reliability metric of interest. This is mostly used at transmission level and is usually less reported by distribution network operators (DNOs) but it is gradually being considered by regulators for an optimal system performance due to its value in the quantification of customer satisfaction. 
This can be seen in a few European countries where ENS is currently assessed at distribution level such as Norway and Romania [6]. ENS is also widely used in the literature for network reliability assessment e.g. [22, 32]. As the power grid becomes more complex, this index will have to inevitably be considered as a benchmark for the performance assessment of DNOs.

\subsubsection{Calculation of the Network Reliability Model}

The first step in the implementation of the MOR procedure is the definition of $x$ and $y$ and the calculation of the matrices $A$ and $C$ in the dynamical system (1) describing the reliability of the network. From Section 4.1, $x(t)$ denotes the probability of each possible combination of reliability states of the system components at time $t$. The matrix $A$ describes the evolution of $\mathrm{x}$ over time and is obtained from (13), where $\lambda$ and $\mu$ denote respectively the failure and repair rates of the PCs and can be obtained from available published reliability data. The output $y(t)$ is assumed to be equal to the PNS of the system at time $t$, so that its integral over time returns the chosen reliability index, ENS. Since a single output is considered, the matrix $C$ has dimensions $1 \times n$ and its $\mathrm{k}^{\text {th }}$ component corresponds to the power not supplied in the $\mathrm{k}^{\text {th }}$ reliability state of the system components considered in the state $x$. For example, $k=1$ denotes the scenario with all system components in the UP state and therefore the associated value $c_{1}$ will be equal to 0 . Conversely, since $k=n$ corresponds to the case with all components in the DOWN mode, the associated value $c_{n}$ will be equal to the total power not supplied in this scenario. The calculation of $C$ in networks with complex topology requires the use of power simulation software PSS ${ }^{\circledR} \mathrm{E}$ (automated using Python), as power flow assessments must be used to determine the amount of power not supplied at each load point (LP), for each reliability state. Algorithm 1 summarises the steps used to construct matrix $C$ in all the analyses presented.

\subsubsection{Calculation of the Reduced-order Model}

The MOR methodology is summarised as follows: develop a complete statespace representation (1) of a network reliability model; use system Gramians to determine which states contain the most useful 'information content' with respect to the selected reliability index (ENS); neglect the states with lower impact; finally, calculate a state-space representation of the reduced-order model that can adequately approximate the original system. The first step to perform the MOR on the original network model is to list the $N$ repairable 


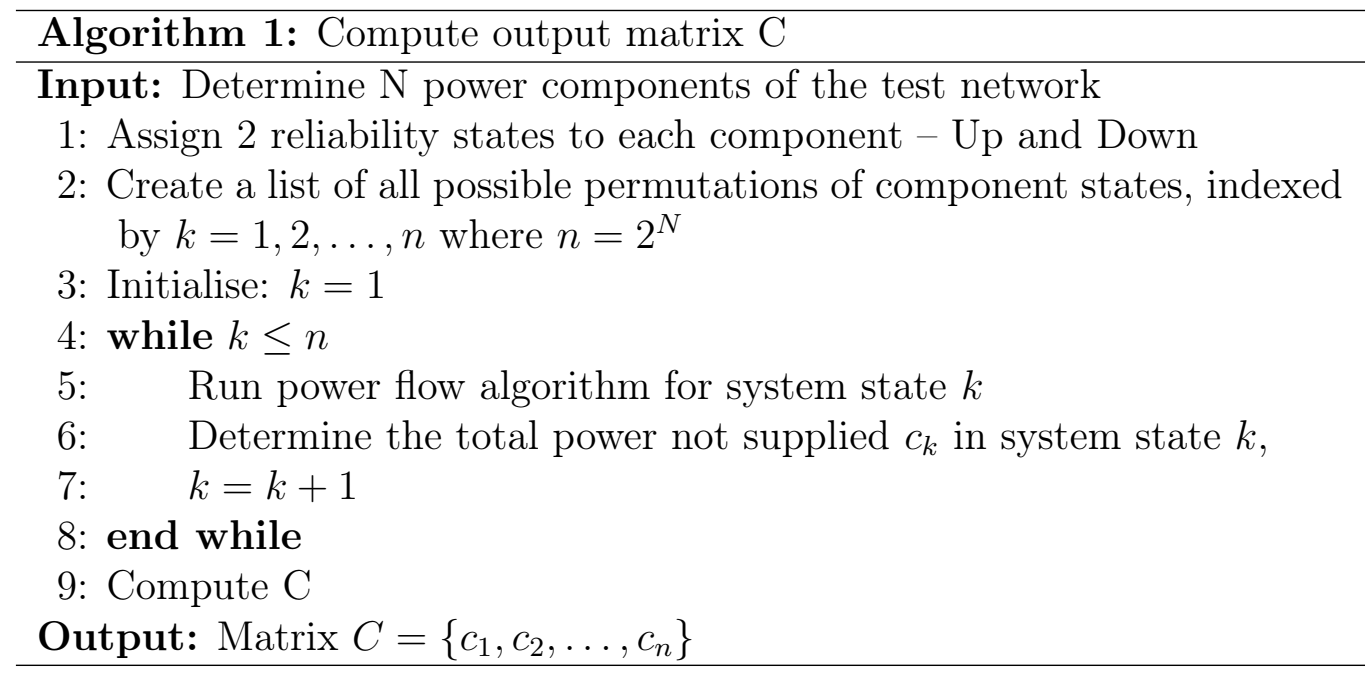

PCs that comprise the network and calculate the resulting failure and repair rates ( $\lambda$ and $\mu$, respectively) from available reliability statistics. For simulation purposes, system (1) and all the associated systems are converted from continuous to discrete time, adequately rescaling the transition rates $\lambda$ and $\mu$ according to the chosen simulation time-step, for example, $\Delta t=1$ hour. Furthermore, matrix $C$ is obtained using Algorithm 1.

It is important to appreciate that $A$ in (13) has a rank of $n-1$, where $n$ is the number of system states [3]. This follows from the chosen probabilistic description of the system, as the sum of probabilities for all the possible reliability states must always be equal to 1 i.e. the sum of columns in ma$\operatorname{trix} A$ (i.e. the derivative of the total probability) must be equal to zero. This means that each column of A has zero sum (i.e. the sum over all the elements of each column is zero) and therefore one row can be written as the total sum (with changed sign) of the others. It follows that, a single state $x_{n}$ can be neglected in the analysis and simply calculated ex-post as the sum of 1 minus the probability of the other states, modifying the $A$ and $C$ matrices accordingly. For this study, the removed system state is the one representing the probability of all PCs being in the UP state. The next step is to determine the transformation matrix $T$ using (10) and then truncating (7) according to (8). This allows for a model reduction of the reduced form of the matrix $A$ (which is full rank) from order $n-1$ to $r-1$. Finally, the initially removed state is added back to the reduced model exploiting the aforementioned property of the sum of the derivatives $\dot{x}(t)$ and obtaining 
the new linear system as represented in (2). Notably, studies in [29] show that the calculation of the system observability Gramian $Q$ in (4) using the new full rank state matrix $A$ ensures that system stability is preserved when MOR is done using truncation.

\subsection{Method Limitations}

The proposed methodology represents a significant step towards a simplified and accurate analysis of complex networks. As demonstrated in the case studies of Section 5, the presented MOR approach allows for a faster reliability assessment with a minimum impact on accuracy. Nevertheless, the proposed approach still exhibits some limitations that will be tackled in future work. In particular, with the current formulation, the number of PCs of the original system that can be modelled is limited by hardware constraints. The two main computational-memory bottlenecks arise from building the state transition matrix $A$ (13) and obtaining the system Gramians (4) by solving computationally expensive Lyapunov equations [29, 31]. These issues were tackled by developing ad-hoc programming solutions and adopting the matrix equation sparse solver (MESS) toolbox [34] for a more efficient resolution of high-order Lyapunov equations. In future work, different techniques will be explored to obtain a faster computation of the relevant Gramian matrices, allowing for the simulation of larger systems. These techniques will exploit the low-rank property for solutions of large-scale, sparse Lyapunov equations [29] e.g. methods based on the Arnoldi process [35, 36] and Krylov subspace methods [36, 37]. A distributed system reduction will also be investigated, deriving the simplified grid model as a collection of interconnected smaller systems, each obtained with the MOR approach presented in this work.

Furthermore, peak demand profiles are utilised at network LPs in addition to the use of constant $\mathrm{PC}$ failure and repair rates used to calculate matrix $A$ in (13). However, further work will integrate time-varying demand profiles as well as time-variant failure rates that account for the PC's lifecycle [38, 39]. These model extensions will be implemented by utilising alternative MOR techniques presented in the literature for time-variant dynamical systems, such as the ones in $[40,41]$. These improvements will be accompanied by the incorporation of different load sectors (residential, industrial and commercial) into the network models to allow for a more accurate quantification of the impact of each system state based on the nature of the load supplied and the time during which network interruptions (leading to ENS) occur. 
Also, for MCS analyses, the current methodology only returns models of order $r=2$. Higher values of $r$ result in system matrices $A$ in (13) that are not in Metzler form. Therefore, the associated system lacks the Markov property and cannot be simulated with MCS methods. Future research will test new methods for the approximation of Metzler matrices. Work in $[42,43]$ investigated this aspect but the proposed methods were not directly applicable to reliability studies because they focused on the stability of the resultant Metzler matrix rather than its Markovian properties.

Finally, the frequency and duration of interruptions are not explicitly included in the chosen state-space representation (1) of the grid reliability. This means that reliability metrics - SAIFI and SAIDI can be calculated ex-post with MCSs but cannot be used as relevant metrics over which the approximation error of the proposed MOR procedure is minimised. However, SAIFI and SAIDI represent two fundamental indices in the evaluation of network reliability and therefore, in order to explicitly consider them in the MOR procedure, the current model will be expanded in future works, for example including additional states in (13) that keep track of the failure times of the different power components. Nonetheless, the analysis is still capable of demonstrating the effectiveness of the proposed MOR methodology, as the key aspect of its validation lies in comparing system outputs of the original vs. reduced-order system [29].

\section{Results and Discussions}

Relevant case studies are considered to evaluate the performance of the proposed MOR technique and its capability to generate accurate reduced models of system reliability. This work assumes an ideal operation of conventional generators in its network reliability assessment and focuses on the failure/repair behaviour of PCs at a transmission and distribution level. These include underground cables, overhead lines, transformers, protection devices, capacitor banks and busbars. Also, all analyses were carried out by using the high-performance computing (HPC) facilities at the University of Bath, UK. The particular hardware used has the following specifications: dual socket

Intel Ivybridge nodes with E5-2650v2 processors, 2.6GHz with 8 cores, and $512 \mathrm{~GB}$ of memory [44]. 


\subsection{Illustration of MOR Functionality}

An example of the proposed modelling approach and the associated MOR methodology is presented for the simple network in Fig. 3. This network model is converted to state-space representation (1) by first defining the state vector $x$ and output $y$. State $x$ denotes the probability of each possible combination of reliability states of the system PCs (i.e. buses and lines) at time $t$. The associated state matrix $A$ in (13) is calculated by using failure and repair rates of all system PCs and has a dimension of $n \times n$ where $n=2^{|P C s|}$ is the order of the system. The output $y$ denotes the system output in terms of the total PNS to the two load points. It is a linear combination of entries in Matrix $C$ that correspond to the PNS associated to each system reliability state. For example, $x_{1}$ denotes the probability that all PCs are in the UP state and all the required power is being supplied, implying $c_{1}=0$. Conversely, since $x_{2}$ represents the probability that all PCs are in the UP state except bus B2, then the associated entry $c_{2}$ in matrix $\mathrm{C}$ will be equal to the power demand that is not being supplied in this case at bus B2, i.e. $c_{2}=10$.

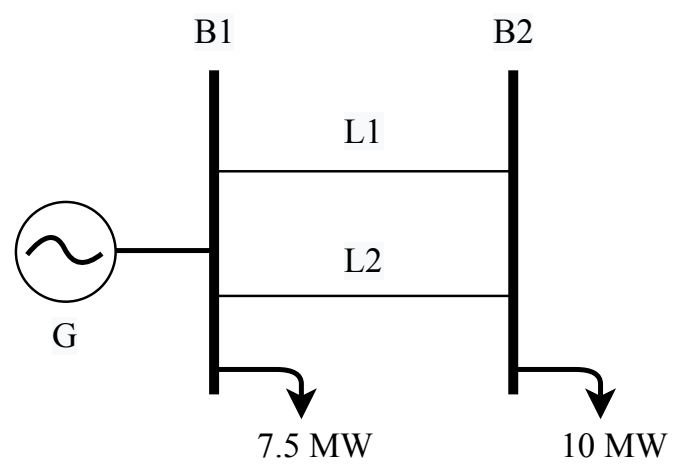

Figure 3: Test system with 4 components (system order $n=2^{4}=16$ ).

Having asserted the meaning of $x(\cdot)$ and $y(\cdot)$ in the context of network reliability, as well as having calculated all system descriptor matrices, the system in Fig. 3 is reduced using the MOR procedure presented. Recalling that this system has no input, the transformation matrix $T$ for the change of coordinates in the original system is obtained using (10) instead of (6). The reduced-order system (2) is then determined by truncating system (5) according to (7)-(8). For a certain order $r$ of the reduced model, the corresponding error bound on the output (i.e. on the chosen reliability index) is given 
by (12). As expected, larger values of $r$ allow to include more information content and therefore achieve higher accuracy.

In Fig. 4, we plot the output of the dynamical system, i.e. the power not supplied which, integrated over time, will equal the system energy not supplied. We recall that this is expressed as $C x(t)$ in (1), where the single component $x_{i}(t)$ of the vector $x(t)$ indicates the probability of being in state $i$ at time $t$. Note that the initial conditions of the system, denoted by $x(0)=$ $x_{0}$, do not affect the steady-state behaviour of the system but only determine the starting point $y(0)=C x(0)$ of the output response. For example, if it is known with probability 1 that all components are UP (working), then the initial PNS will be $0 \mathrm{MW}$ and it will then increase to a steady state value $y_{s s}=C x_{s s}$ over time. Zoomed-in values in Fig. 4 show that the steady state value obtained for PNS $\left(y_{s s}\right)$ is non-zero because the network contains uncontrollable PC failures that result in a small amount of unsupplied power in steady state conditions. Fig. 4 also compares the output (i.e. the PNS index) of the original system with the output of a selection of reduced-order models with $r=2$ and 4 . As expected, the evolution of the PNS of the reduced-order models follows more closely the one of the original system when $r$ is larger and more states are kept in the model. Indeed, the system behaviour for the reduced-order system with only 4 states (MOR-4 states) indicates no appreciable difference in the output of the reduced-order models and that of the original system.

The analysis on this simple example is extended further by considering each possible order $r$ for the MOR and quantifying the resulting reliability error as follows:

$$
\text { Error }_{r}=\frac{\left\|\hat{y}_{r}-y\right\|_{2}}{\|y\|_{2}} \times 100
$$

where $r$ is the order of the reduced system and $\hat{y}_{r}$ is the resulting output, $\|\cdot\|_{2}$ is the L2 norm, and the error value is in per cent. In this case, $y$ and $\hat{y}_{r}$ are vectors representing the outputs of the original and reduced-order system, evaluated at discretised time instants. Fig. 5 compares the accuracy of the reduced model (i.e. percentage error in the evolution of the system's PNS) in Fig. 5(a) with the system HSVs $\sigma_{i}$ in Fig. 5(b). Recall that the HSVs associated to the system states that are not included in the new reduced model quantify the upper bound on the approximation error of the analysis, 


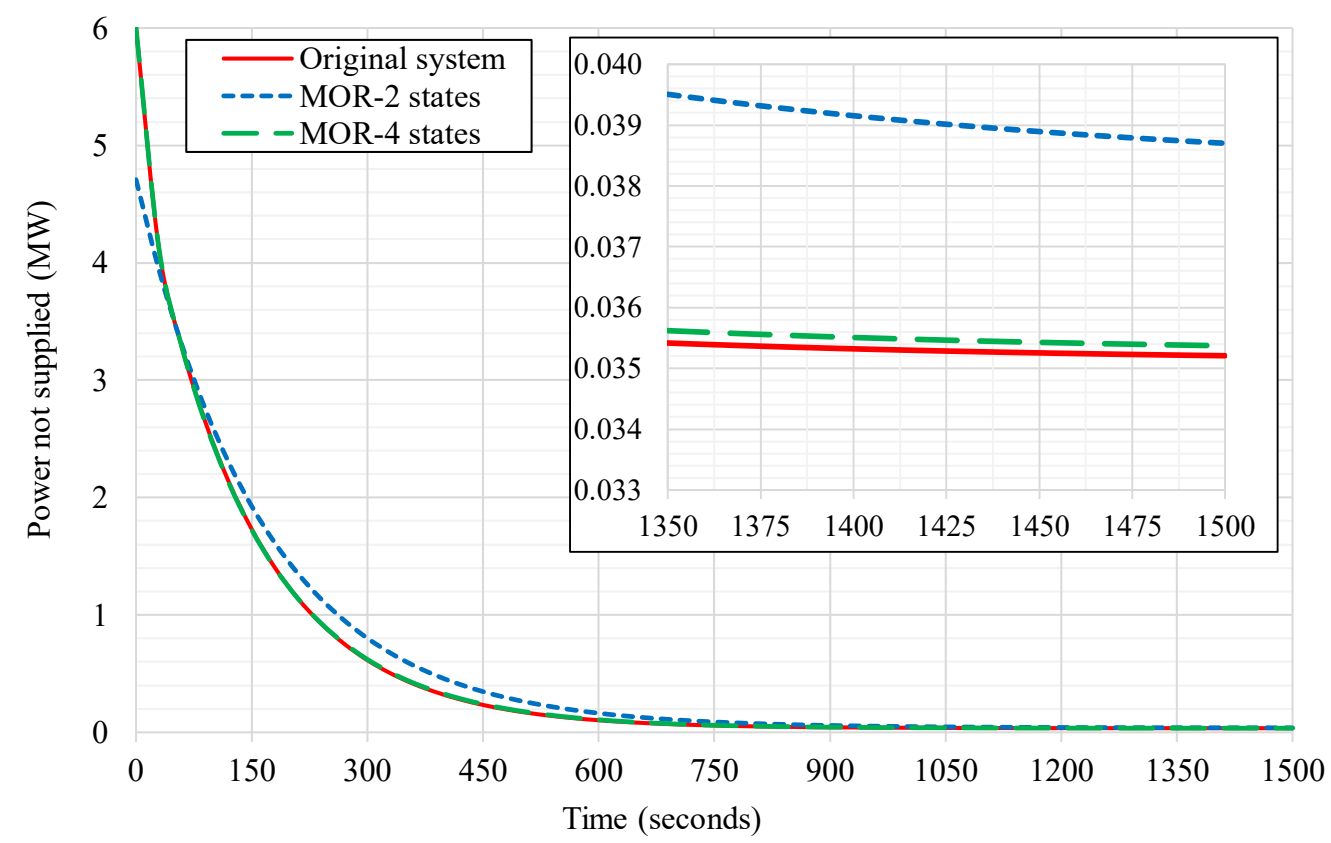

Figure 4: Evolution over time of PNS for the original system and reduced models with $r=2$ and 4 .

according to (12). Using Fig. 5(a) for comparison, there is a clear correlation between the system error attained with a reduced system of a certain order $r$ and the HSVs associated to the number of system states considered in the reduced model.

\subsection{MOR Reliability Performance Evaluation}

To assess the performance of the proposed MOR technique, time-sequential MCSs are carried out on the original and reduced models to compare the required simulation time and quantify the accuracy of the reliability assessment. The MCS evaluates the annual ENS of the test systems by using a time step of 1 hour [45]. Moreover, to obtain acceptable index accuracy in all considered MCSs, and accurately compare the computational burden of both the original and reduced models, the total simulation period (or number of MCS samples) is based on achieving set thresholds for the coefficient of variation $(\mathrm{CoV})[45,46]$. The threshold value for the $\mathrm{CoV}$ is set to $0.2 \%$, which is well below the typical tolerance level for ENS as given for example in [47] for different reliability indices. 


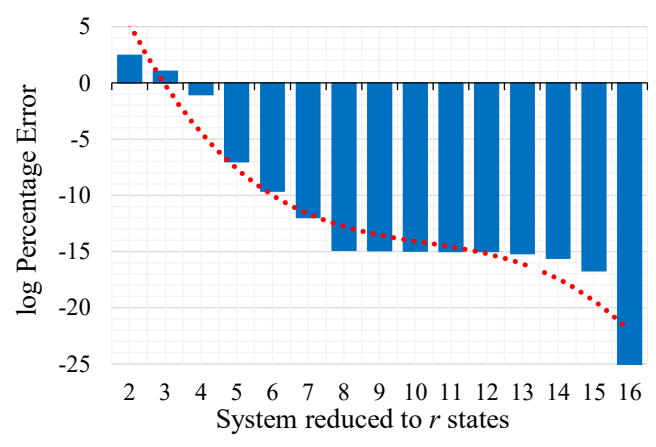

(a) Percentage Error

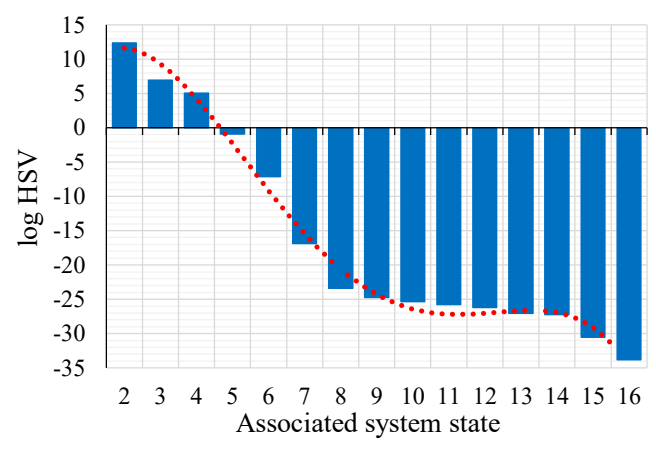

(b) HSVs

Figure 5: Comparison between the accuracy of the reduced model and the HSVs of the original system.

\subsubsection{Test System with 4 PCs}

The test system in Fig. 3 is composed of 4 PCs, each with only 2 possible reliability states (UP/DOWN), and its dimension is equal to $2^{4}$. Following from the discussion in Section 4.2, this original system is reduced to 2 states using MOR and the ENS is evaluated in the two cases. Results are presented in Table 1 where the performance evaluation of MOR is compared with two other methods. As described in Section 2, using traditional network reduction (TNR) techniques, the original system is simplified to a single equivalent element by systematically combining appropriate series and parallel branches of the reliability network. Then, the reliability of the remaining equivalent element equals the reliability of the original network [3] and will exhibit the same unavailability $U$ (i.e. probability of being in DOWN mode). The relevant equations for reducing a network with 2 repairable PCs in series and parallel configurations are presented in [3]. The other existing method termed AEM [48] calculates the equivalent PC failure rate as the sum of all $\mathrm{PC}$ failure rates, while the equivalent $\mathrm{PC}$ repair rate is the reciprocal of the average of all $\mathrm{PC}$ repair rates. It is important to note that in order to fairly compare the performance of the proposed MOR technique with the two other techniques (TNR and AEM), all 3 reduction methods are used to reduce the original system of 16 states to 2 states (or one equivalent component). The ENS is then calculated in each case to determine the associated error. Moreover, given the fact that the original system is of a relatively small size, computational time reduction results are not presented as the reduction to 2 states in all cases does not offer any significant time saving from the 
original case. Instead, Table 1 focuses on the error obtained when assessing ENS for reduced order networks. Further analyses presented in the next subsections demonstrate the computational time saving achieved using the reduction methodology when applied to larger networks.

Table 1: Reliability performance for the $4 \mathrm{PC}$ test system.

\begin{tabular}{lccl}
\hline Network & $\begin{array}{l}\text { System } \\
\text { States }\end{array}$ & $\begin{array}{l}\text { Average ENS } \\
\text { (MWh/year) }\end{array}$ & $\begin{array}{l}\text { ENS } \\
\text { Error (\%) }\end{array}$ \\
\hline Original & 16 & 307.88 & \\
MOR & 2 & 335.09 & $8.84 \%$ \\
TNR & 2 & 391.76 & $27.25 \%$ \\
AEM & 2 & 504.32 & $63.81 \%$ \\
\hline
\end{tabular}

Table 1 illustrates the advantages of the MOR technique in terms of accuracy of network reliability assessment. The ENS value of the reduced network calculated with the new proposed approach exhibits the lowest error with respect to the original system (8.84\%) when compared to both TNR and AEM techniques. It is important to note that this demonstrates the capability of the proposed MOR method in accounting for dispersed loads during network reduction unlike the case in both TNR and AEM techniques. This results in higher accuracy when using MOR for networks often characterised by highly dispersed loads as shown in the next subsection.

\subsubsection{Roy Billinton Test System}

The MOR approach for reliability analysis is applied to a relevant test case - the Roy Billinton Test System (RBTS) in Fig. 6. This is a composite power system with 11 generator units, 2 generation buses, 4 load buses and 9 transmission lines. The transmission system voltage level is $230 \mathrm{kV}$ and the bus voltage limits are 1.05 p.u. and 0.97 p.u. The total generation capacity is $240 \mathrm{MW}$ while the peak load is $185 \mathrm{MW}$. Bus 1 is assumed to be the slack bus under normal circumstances and the power factor at each bus is unity. The basic bus and transmission line reliability data i.e. failure rates and repair times are obtained from [49]. Based on the aforementioned assumptions, the original RBTS 'offers' a total of 15 PCs (6 buses +9 lines) for this analysis each with only 2 possible reliability states, and its dimension is equal to 215. This original system is reduced to 2 states using MOR and the ENS is evaluated in the two cases. The complexity of this network 
configuration (meshed topology) means that TNR cannot be used to effect model reduction. Therefore, MOR is only compared with the AEM approach in the results presented in Table 2 .

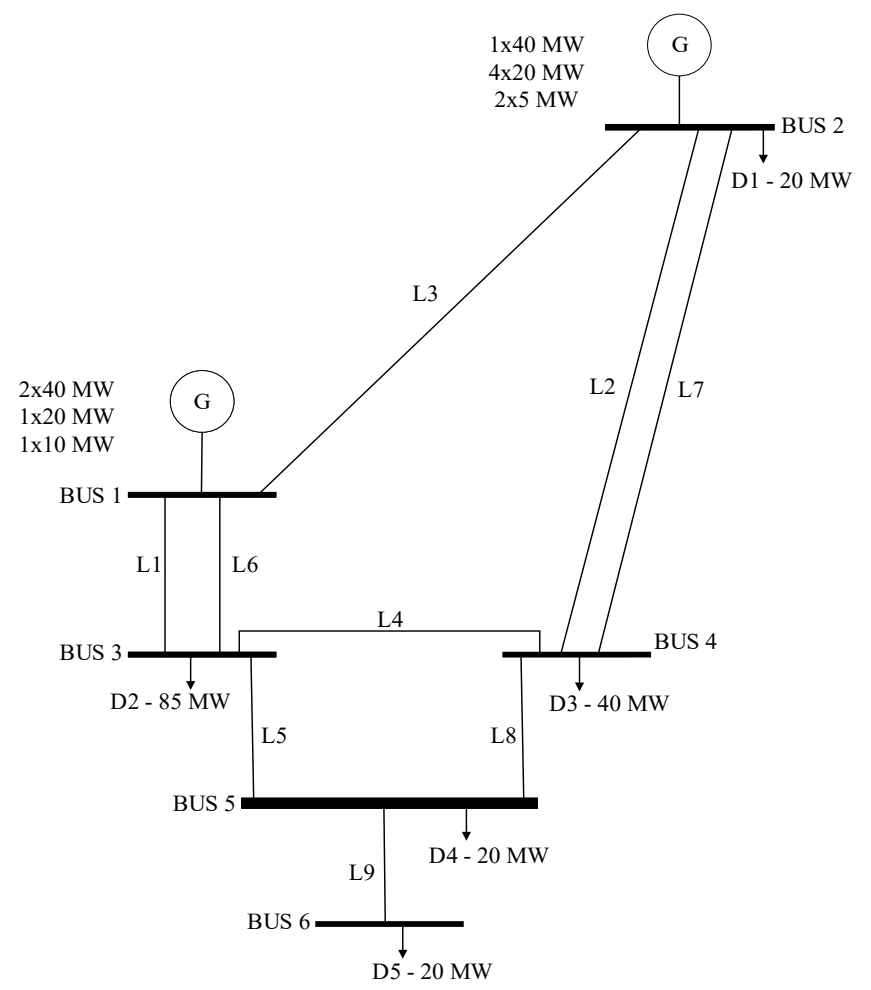

Figure 6: Single line diagram of the original RBTS with 15PCs [49].

Table 2 results illustrate that the reduced-order model obtained with the proposed MOR methodology estimates the ENS index of the RBTS with good accuracy while also providing a significant saving in the total computational time required to complete the reliability assessment. This shows that by constructing a reduced reliability model that only retains the most representative states with the highest 'information content', it is possible to trade much faster resolution times with a minimum impact on the accuracy of the reliability analysis. Table 2 also proves that the performance improvement of the proposed MOR method with respect to the AEM approach is even more significant in meshed networks, which are often endowed with redundant components to facilitate provision of backup supply in case a PC with a parallel operation fails. In the AEM approach, these 'additional' PCs only 
serve to increase the total number of failures and thus result in a $6082.15 \%$ error in ENS calculation compared to only $6.28 \%$ in the MOR case. However, it is evident in Table 2 that there exists a slightly better time saving in computational time with AEM (96\%) as compared to MOR (92.12\%). This is because the time required to perform the order reduction (OR) of the original system to 2 states using MOR is significantly higher than with the AEM approach. Additionally, Table 2 also reveals that the relatively low $\mathrm{CoV}$ threshold $(0.2 \%)$ accounts for the relatively large times required before MCS converges for each simulated network model.

Table 2: Reliability performance for the RBTS with 15PCs.

\begin{tabular}{|c|c|c|c|c|c|c|}
\hline \multirow{2}{*}{ Network } & \multirow{2}{*}{$\begin{array}{l}\text { Average ENS } \\
(\mathrm{MWh} / \text { year) }\end{array}$} & \multirow{2}{*}{$\begin{array}{l}\text { ENS } \\
\text { Error (\%) }\end{array}$} & \multicolumn{3}{|c|}{ Computational Time (s) } & \multirow{2}{*}{$\begin{array}{l}\text { Total Time } \\
\text { Saving (\%) }\end{array}$} \\
\hline & & & OR & MCS & $\mathrm{OR}+\mathrm{MCS}$ & \\
\hline Original & 651.30 & & & 137089.0 & 137089.0 & \\
\hline MOR & 692.20 & $6 . \overline{28} \%$ & $53 \overline{59} .6$ & 5443.4 & 10803.0 & $92.12 \%$ \\
\hline AEM & 40264.56 & $6082.15 \%$ & 0.0 & 5484.2 & 5484.2 & $96.00 \%$ \\
\hline
\end{tabular}

Furthermore, to demonstrate the varying accuracy of the proposed MOR technique when applied to similar models of different size, a sensitivity analysis is performed by varying the size of the original system rather than the order of the reduced model. The original RBTS is simplified by applying standard techniques of reliability block diagrams [3]. This approach is used to obtain three different representations of the RBTS with 15, 12 and 9 PCs, respectively. As an example of the transformations that have been considered, lines 1 and 6 of the network, which connect bus 1 to bus 3 in Fig. 6 , are replaced by a unique equivalent line, whose fail/repair parameters have been obtained using the relevant equations in [3]. Each of the original RBTS models is reduced to $r=2$ states using the proposed MOR technique, followed by time-sequential MCS analyses as described previously. The error in the estimation of the ENS and the computational time saving in the three representations of the RBTS are reported in Fig. 7 as a function of the original system size. As expected, Fig. 7(a) shows that a higher accuracy is achieved when the starting original system is less complex and has a lower number of PCs. Conversely, Fig. 7(b) shows that there is a more consistent reduction of the computational time of the MCS when the 15PCs network is reduced to 2 states, as compared to an identical operation performed on the 9PC network.

Notably, the time saving $(92.12 \%)$ achieved when the 15 PCs network is re- 


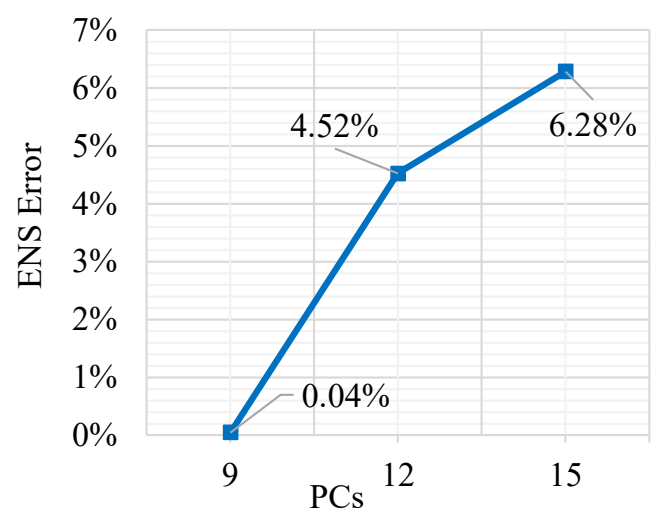

(a) ENS error

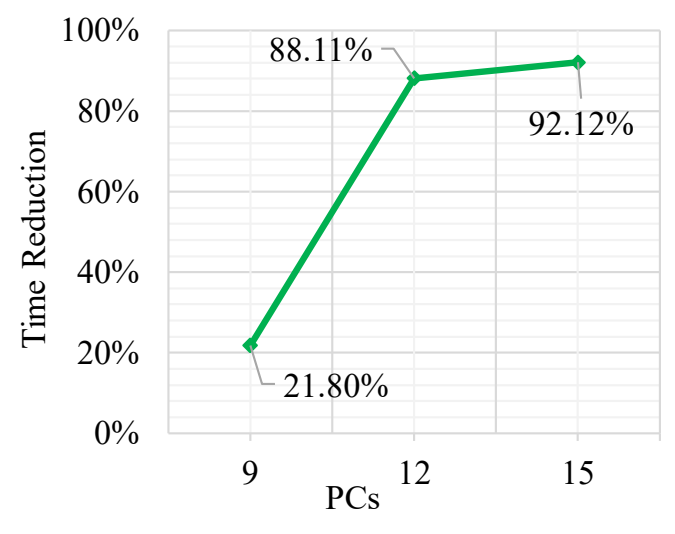

(b) MCS computational time reduction

Figure 7: Trends of MOR accuracy and MCS computational time with respect to network size.

duced is only marginally higher than the saving (88.11\%) when the $12 \mathrm{PCs}$ network is reduced. This is because while the reduced order systems in both cases have similar times for MCS convergence, the time required to perform MOR in the $15 \mathrm{PC}$ case is $5359.6 \mathrm{~s}$ compared to only $35.8 \mathrm{~s}$ in the $12 \mathrm{PC}$ case (and $0.5 \mathrm{~s}$ in the $9 \mathrm{PC}$ case). Nonetheless, the use of the proposed MOR technique results in a significant time saving in all cases and a validation of the expected trends for the sensitivity analysis.

\subsubsection{Generic Medium Voltage Substation}

To further demonstrate the performance of the proposed MOR methodology, it is tested on a generic medium voltage (MV) substation presented in Fig. 8. With respect to the RBTS, which is a meshed network, this network exhibits a radial configuration. Moreover, it only has one aggregate load (and LP) as compared to 5 different LPs in the RBTS. This network, adapted from work in [50,51], consists of two 15 MVA $33 / 11 \mathrm{kV}$ transformers supplying a mainly residential load of 9120 customers. The total average load is measured at 20.7 MW. Downstream the main $11 \mathrm{kV}$ bus, only one equivalent feeder is used to represent the rest of the network, as well as the total aggregate load. The sensitivity analysis for this test case is also performed using the aggregation methods summarised in [3], resulting in 4 'versions' of this network (10, 9, 7 and 4 PCs respectively). Table 3 shows the varying number of types of components for each network. The reliability data used for these PCs are 
obtained from [51].

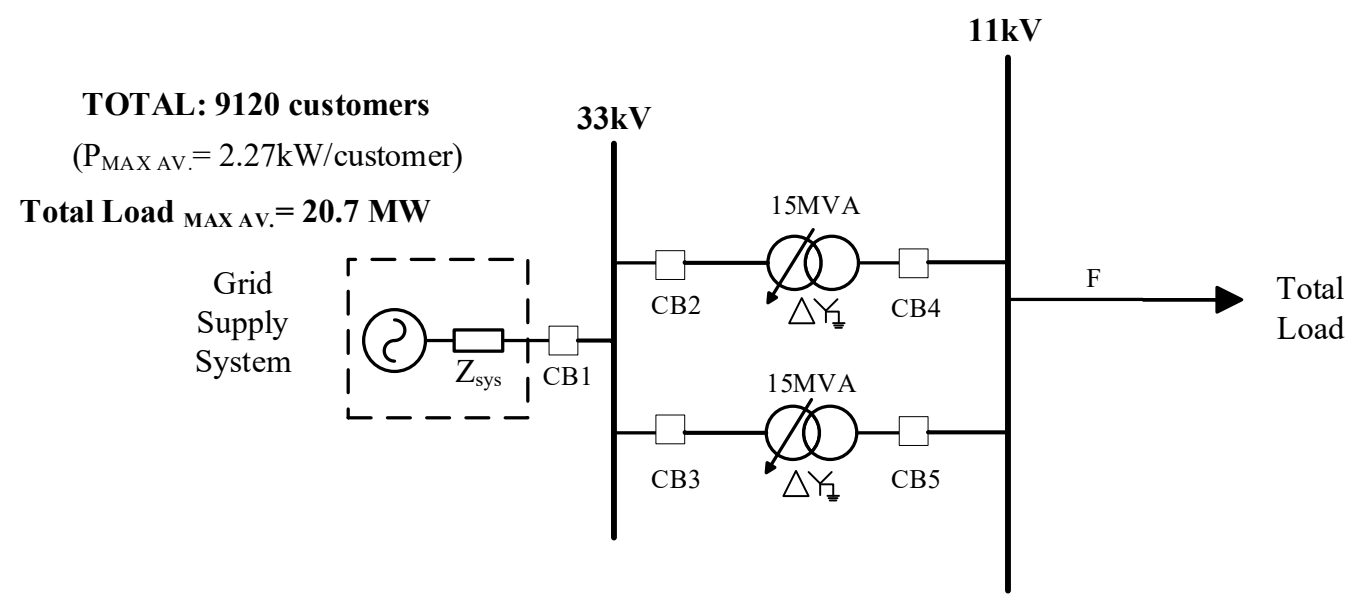

Figure 8: Generic MV substation model - 10PCs.

Table 3: Generic MV substation configurations.

\begin{tabular}{lcccc}
\hline Type of & \multicolumn{4}{c}{ Original } \\
\cline { 2 - 5 } Compotwont & $\mathbf{4}$ & $\mathbf{7}$ & $\mathbf{9}$ & $\mathbf{1 0}$ \\
\hline Buses & 2 & 2 & 2 & 2 \\
Transformers & 2 & 2 & 2 & 2 \\
Circuit breakers & 0 & 3 & 5 & 5 \\
Feeders & 0 & 0 & 0 & 1 \\
\hline
\end{tabular}

Fig. 9 compares the results obtained when each of the networks (4PC-10PC) is reduced to $r=2$ states. As shown in Fig. 9(a), the ENS error increases when larger networks (i.e. more PCs) are considered. This represents an anticipated result since a reduction to only 2 states will be unable to capture the most relevant dynamics if the original system is too large and complex. Conversely, Fig. 9(b) proves that the saving in the total computational time required for analyses is much larger when MOR is applied to a larger network (10PCs) as compared to a smaller one (4PCs). Notably, in all cases the computational time is mostly taken up by MCS, as the times required to perform MOR are only 0.05, 0.15, 0.39 and 1.44 seconds for the 4, 7, 9 and 10 PC systems respectively. This results in a negligible impact of MOR-time 
on the total computational time required to perform a reliability assessment for this network configuration.

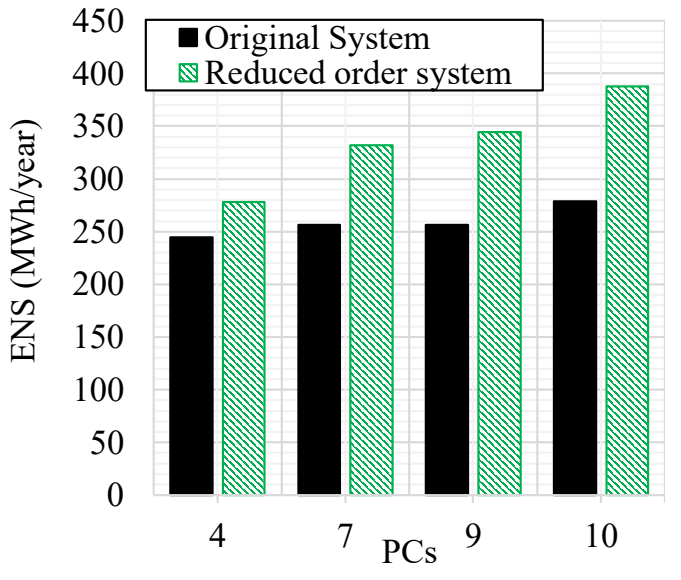

(a) ENS

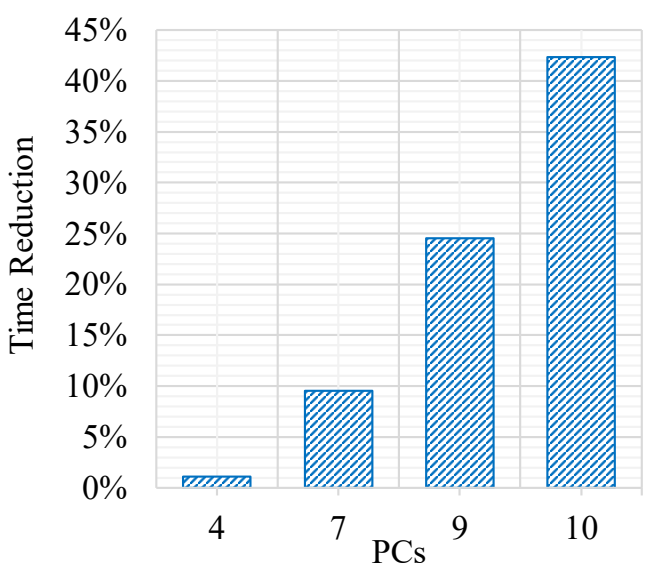

(b) Total Computational time

Figure 9: Trends of reliability accuracy and computational time of the MOR methodology with respect to the original size, for the generic MV substation presented in Fig. 8.

\subsubsection{Medium Voltage Distribution Network}

The substation model presented in Fig. 8 is expanded into a small-scale distribution network as shown in Fig. 10 to further demonstrate the applicability of the proposed method. This network is adapted from a typical underground MV network for urban areas as presented in [51]. Accordingly, it presents a meshed configuration where normal network operation is supported with another supply point e.g., another primary substation or a "reflection centre". The reflection centre guarantees the supply of all feeders connected from both ends of the network.

It is important to note that Fig. 10 is a scaled down version of the actual representation of an urban MV network due to the aforementioned hardware constraints in the implementation of MOR for large systems. The full network is typically designed for a maximum of six $11 \mathrm{kV}$ feeders and ten $11 / 0.4 \mathrm{kV}$ distribution transformers from each $11 \mathrm{kV}$ feeder. This is in addition to the use of necessary protection devices such as fuses and circuit breakers. For this analysis, the distribution network in Fig. 10 presents a total of 14 components (2 buses, 6 transformers, 4 underground cables and 2 switches). Furthermore, the network is modelled to have 4 LPs each 


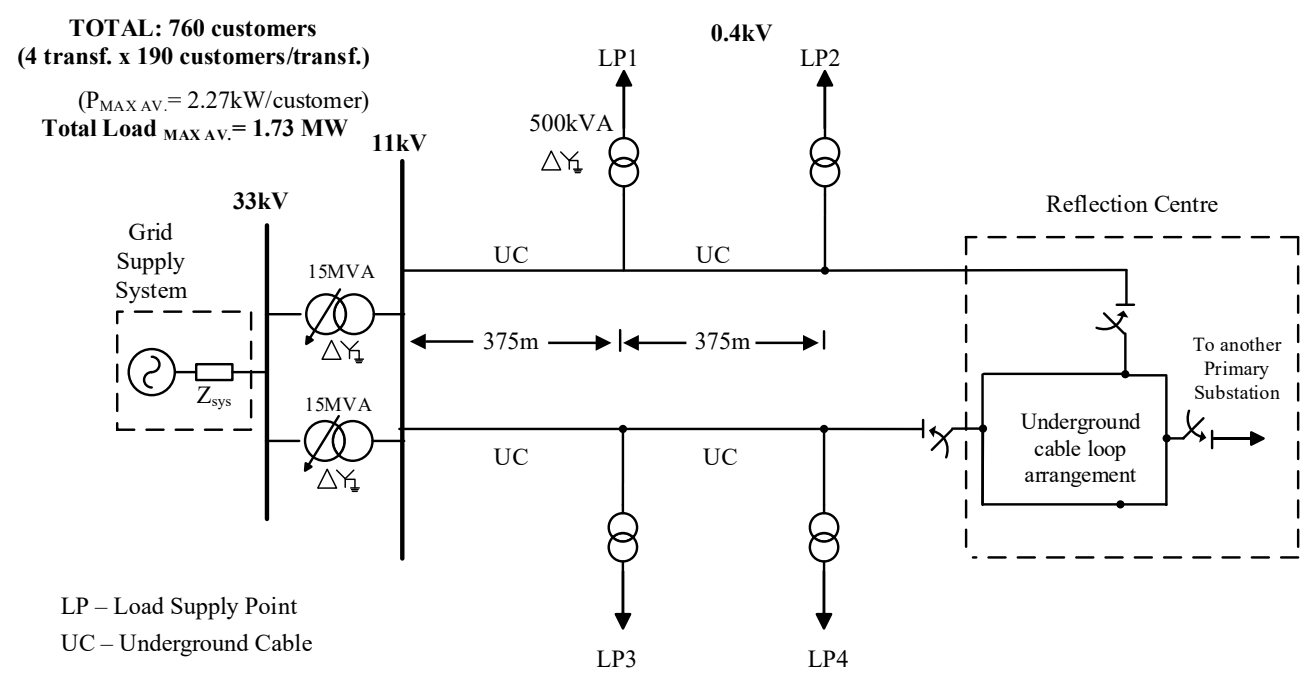

Figure 10: Small-scale distribution network model - 14PCs.

supplying a total of 190 customers through a $500 \mathrm{kVA} 11 / 0.4 \mathrm{kV}$ distribution transformer. The basic reliability data used are obtained from [51]. For the results presented in Table 4, the original distribution network with $2^{14}$ states is reduced to 2 states using the proposed MOR approach. As seen with the RBTS network, it is not possible to reduce this distribution network using the TNR approach because of the network topology. Table 4 demonstrates that the reduced order model obtained using the proposed MOR approach results in a good accuracy for the estimated ENS-index (4.15\%) while providing a significant saving in the required computational time.

Table 4: Reliability performance for the distribution network.

\begin{tabular}{|c|c|c|c|c|c|c|}
\hline \multirow{2}{*}{ Network } & \multirow{2}{*}{$\begin{array}{c}\text { Average ENS } \\
(\mathrm{kWh} / \text { year })\end{array}$} & \multirow{2}{*}{$\begin{array}{l}\text { ENS } \\
\text { Error (\%) }\end{array}$} & \multicolumn{3}{|c|}{ Computational Time (s) } & \multirow{2}{*}{$\begin{array}{l}\text { Total Time } \\
\text { Saving (\%) }\end{array}$} \\
\hline & & & OR & MCS & $\mathrm{OR}+\mathrm{MCS}$ & \\
\hline Original & 263.68 & & & 76239.0 & 76239.0 & \\
\hline MOR & 274.62 & $4.15 \%$ & 1611.6 & 4922.9 & 6534.5 & $91.43 \%$ \\
\hline
\end{tabular}

\subsection{Integration of $P V$ and Storage Technologies}

The capability of the proposed MOR methodology to accommodate PV and ES in the simplified reliability models is now demonstrated. For this purpose, four distinct scenarios (presented in Table 5) are considered for the RBTS 
12PC network. The SC1 (base case) scenario considers no PV or ES, while $\mathrm{SC} 2 \mathrm{~A}$ and $\mathrm{SC} 2 \mathrm{~B}$ scenarios represent the addition of PV to the network with different penetration levels. In this case, the PV generation is modelled as a constantly available power source whose power output is equal to the average of a typical PV generation profile. This is considered a good approximation of a realistic scenario if one assumes that the faults of PCs are uniformly distributed over time. Further work will ensure that the intermittent nature of the supply from PV, as well as the daily and seasonal cycles, can be added into the analysis.

Finally, SC3 includes ES resources, which are assumed to be locally available only to LPs D4 and D5 (combined 40MW load). Given that these loads are the furthest from the main supply, it is expected that the use of ES will greatly enhance not only the ENS of the customers at LPs D4 and D5 but also the average system ENS. This ES configuration is designed to supply energy in the event of a fault occurrence that causes outage to either D4 or D5, and to completely alleviate the effect of faults at these loads. Based on previous work from the authors, further analyses will integrate other parameters such as energy price signals, state of battery charge, solar irradiance, or timevariant demand to the state and capacity of the ES system [50].

Table 5: Reliability performance with PV and ES.

\begin{tabular}{llccl}
\hline ID & Scenario & \multicolumn{2}{c}{ Average ENS (MWh/year) } & ENS \\
\cline { 3 - 4 } & & $\begin{array}{c}\text { Original system } \\
\text { (12PCs) }\end{array}$ & $\begin{array}{c}\text { Reduced-order } \\
\text { system }\end{array}$ & \\
\hline SC1 & Base case & 651.87 & 681.33 & $4.52 \%$ \\
SC2A & PV 25\% & 488.90 & 511.11 & $4.54 \%$ \\
SC2B & PV 50\% & 325.93 & 340.74 & $4.54 \%$ \\
SC3 & ES & 319.85 & 335.43 & $4.87 \%$ \\
\hline
\end{tabular}

As expected, results in Table 5 show that as PV penetration increases, the ENS to customers is progressively reduced. Furthermore, ES implementation almost halves the system ENS with respect to the base case, where the LPs D4 and D5 present poor reliability. Overall, the reduction of the system to 2 states in each scenario using MOR resulted in an error of only about $4.5 \%$ (Table 5). This demonstrates the capability of the MOR methodology to reduce the computational time of reliability analyses while accurately 
representing the impact of PV and ES on network reliability.

\section{Conclusions and Further Work}

This paper presents a novel application of MOR techniques for reliability analysis of distribution networks. The proposed analytical methodology, based on balanced truncation, allows to determine reduced network models with lower complexity and faster simulation times while minimising the resulting error on reliability metrics. The practical implementation of the algorithm has been described in detail and relevant case studies have been presented to demonstrate the capability of the proposed approach to capture the most important reliability dynamics of the original networks and provide simplified models that describe with good approximation the reliability of the original system. As expected, these simplified models guarantee an appreciable saving in the computational time required to perform reliability analyses. The case studies also demonstrate the capability of the proposed approach to assess the impact of PV and ES on the considered reliability metrics. The methodology developed in this paper is the first step towards a utilisation of MOR techniques for reliability assessment of power networks. The improvements suggested in the further work will allow for use of the proposed MOR technique in networks characterised by higher complexity and the substantial penetration of new technologies e.g. demand response, storage, electric vehicles.

Further work will enhance the proposed methodology by including dynamic PC failure rates and time-varying demand profiles. This will be in addition to the use of a minimum load curtailment model in the computation of the output matrix $C$ to ensure that network control actions for minimising the impact of outages to customers are included in the analysis. This inclusion will also be useful to further demonstrate the capability of PV and storage technologies to relieve network constraints and provide ancillary services. Moreover, the effect of cloud transients on PV generation and the evolution of the state of charge of the ES devices will be incorporated in future analyses for a more realistic modelling and analysis of the impact of these technologies on the network reliability. The dependence on computational requirements will also be addressed to enable the modelling of larger and more complex systems. This will include investigating new methods for the approximation of Metzler matrices that preserve their Markovian properties to allow for 
larger orders $(r)$ of the reduced system. An alternative will also be to compare the proposed approach with other MOR approaches e.g. moment-matching. Finally, the methodology will be expanded to accommodate more reliability indices, such as those measuring frequency and duration of interruptions, as well as providing probability density functions to further describe the variation of these indices for given systems as these are key advantages in using MCS analysis for reliability analysis that the reduced system should be able to accurately illustrate. This will represent an important further step towards the application of this methodology in a practical context.

\section{Declaration of Competing Interest}

The authors declare no conflicts of interest.

\section{Acknowledgements}

This research made use of the Balena High Performance Computing (HPC) Service at the University of Bath.

\section{References}

[1] S. M. Ashraf, B. Rathore, S. Chakrabarti, Performance analysis of static network reduction methods commonly used in power systems, in: 2014 Eighteenth National Power Systems Conference (NPSC), IEEE, Guwahati, India, 2014, pp. 1-6. doi:10.1109/NPSC.2014.7103837.

URL http://ieeexplore.ieee.org/lpdocs/epic03/wrapper.htm? arnumber $=7103837$

[2] P. Fortenbacher, T. Demiray, C. Schaffner, Transmission Network Reduction Method Using Nonlinear Optimization, in: 2018 Power Systems Computation Conference (PSCC), IEEE, Dublin, Ireland, 2018, pp. 1-7. arXiv:1711.01079, doi:10.23919/PSCC.2018.8442974.

URL https://ieeexplore.ieee.org/document/8442974/

[3] W. Li, Risk Assessment of Power Systems: Models, Methods, and Applications: Second Edition, Vol. 9781118686, John Wiley \& Sons, Inc., Hoboken, NJ, USA, 2014. doi:10.1002/9781118849972.

URL http://doi.wiley.com/10.1002/9781118849972 
[4] R. Billinton, R. N. Allan, Reliability Evaluation of Power Systems, 2nd Edition, Springer US, Boston, MA, 1996. doi:10.1007/978-1-4899-18604.

URL http://link. springer. com/10.1007/978-1-4899-1860-4

[5] I. Hernando-Gil, B. Hayes, A. Collin, S. Djokić, Distribution network equivalents for reliability analysis. Part 1: Aggregation methodology, in: 2013 4th IEEE/PES Innovative Smart Grid Technologies Europe, ISGT Europe 2013, IEEE, Lyngby, Denmark, 2013, pp. 1-5. doi:10.1109/ISGTEurope.2013.6695450. URL http://ieeexplore. ieee.org/document/6695450/

[6] CEER, 6th CEER benchmarking report on the quality of electricity and gas supply, Tech. rep. (2016).

URL https://www.ceer.eu/documents/104400/-/-/ d064733a-9614-e320-a068-2086ed27be7f

[7] N. D. Tleis, Power Systems Modelling and Fault Analysis, 1st Edition, Elsevier, 2008. doi:10.1016/B978-0-7506-8074-5.X5001-2.

URL https://linkinghub.elsevier.com/retrieve/pii/ B9780750680745X50012

[8] Y. Jiang, N. Acharya, Y. Pan, Model reduction for fast assessment of grid impact of high penetration PV, in: 2017 19th International Conference on Intelligent System Application to Power Systems, ISAP 2017, IEEE, 2017, pp. 1-6. doi:10.1109/ISAP.2017.8071384.

URL http://ieeexplore. ieee.org/document/8071384/

[9] M. Todinov, Methods for Analysis of Complex Reliability Networks, in: M. T. Todinov (Ed.), Risk-Based Reliability Analysis and Generic Principles for Risk Reduction, Elsevier, Oxford, 2007, pp. 31-58. doi:10.1016/b978-008044728-5/50003-2.

URL https://linkinghub.elsevier.com/retrieve/pii/ B9780080447285500032

[10] IEEE Guide for Electric Power Distribution Reliability Indices (2012). doi:10.1109/IEEESTD.2012.6209381.

URL https://ieeexplore.ieee.org/document/6209381 
[11] A. Antoulas, Approximation of Large-Scale Dynamical Systems: An Overview, IFAC Proceedings Volumes 37 (11) (2004) 19-28. doi:10.1016/S1474-6670(17)31584-7.

URL https://linkinghub.elsevier.com/retrieve/pii/ S1474667017315847

[12] C. M. Rergis, R. J. Betancourt, A. R. Messina, Order Reduction of Power Systems by Modal Truncated Balanced Realization, Electric Power Components and Systems 45 (2) (2017) 147-158. doi:10.1080/15325008.2016.1248252.

URL https://www.tandfonline.com/doi/full/10.1080/15325008. 2016.1248252

[13] L. Nechak, H. F. Raynaud, C. Kulcsár, Model order reduction of random parameter-dependent linear systems, Automatica 55 (2015) 95-107. doi:10.1016/j.automatica.2015.02.027.

URL https://linkinghub.elsevier.com/retrieve/pii/ S0005109815000898

[14] A. K. Prajapati, R. Prasad, A New Model Reduction Method for the Linear Dynamic Systems and Its Application for the Design of Compensator, Circuits, Systems, and Signal Processing 39 (5) (2020) 2328-2348. doi:10.1007/s00034-019-01264-1.

URL http://link.springer.com/10.1007/s00034-019-01264-1

[15] X. Lan, H. Zhao, Y. Wang, Z. Mi, Nonlinear power system model reduction based on empirical gramians, in: 2016 IEEE International Conference on Power System Technology, POWERCON 2016, IEEE, Wollongong, NSW, Australia, 2016, pp. 1-6. doi:10.1109/POWERCON.2016.7754074.

URL http://ieeexplore. ieee.org/document/7754074/

[16] D. Osipov, K. Sun, Adaptive nonlinear model reduction for fast power system simulation, IEEE Transactions on Power Systems 33 (6) (2018) 6746-6754. doi:10.1109/TPWRS.2018.2835766.

URL https://ieeexplore.ieee.org/document/8357933/

[17] J. Qi, J. Wang, H. Liu, A. D. Dimitrovski, Nonlinear Model Reduction in Power Systems by Balancing of Empirical Controllability and Observability Covariances, IEEE Transactions on Power Systems 32 (1) (2017) 
114-126. doi:10.1109/TPWRS.2016.2557760.

URL http://ieeexplore.ieee.org/document/7464373/

[18] H. S. Zhao, N. Xue, N. Shi, Nonlinear dynamic power system model reduction analysis using balanced empirical Gramian, in: Applied Mechanics and Materials, Vol. 448-453 of Applied Mechanics and Materials, Trans Tech Publications Ltd, 2014, pp. 2368-2374. doi:10.4028/www.scientific.net/AMM.448-453.2368.

URL https://www. scientific.net/AMM. 448-453.2368

[19] S. S. Mohseni, M. J. Yazdanpanah, A. Ranjbar Noei, Model Reduction of Nonlinear Systems by Trajectory Piecewise Linear Based on OutputWeighting Models: A Balanced-Truncation Methodology, Iranian Journal of Science and Technology - Transactions of Electrical Engineering 42 (2) (2018) 195-206. doi:10.1007/s40998-018-0058-4.

URL http://link. springer. com/10.1007/s40998-018-0058-4

[20] F. D. Freitas, J. Rommes, N. Martins, Gramian-based reduction method applied to large sparse power system descriptor models, IEEE Transactions on Power Systems 23 (3) (2008) 1258-1270. doi:10.1109/TPWRS.2008.926693.

URL http://ieeexplore. ieee.org/document/4558425/

[21] Y. G. I. Acle, F. D. Freitas, N. Martins, J. Rommes, Parameter Preserving Model Order Reduction of Large Sparse Small-Signal Electromechanical Stability Power System Models, IEEE Transactions on Power Systems 34 (4) (2019) 2814-2824. doi:10.1109/TPWRS.2019.2898977.

URL https://ieeexplore.ieee.org/document/8640061/

[22] W. Liu, D. Guo, Y. Xu, R. Cheng, Z. Wang, Y. Li, Reliability assessment of power systems with photovoltaic power stations based on intelligent state space reduction and pseudo-sequential monte carlo simulation, Energies 11 (6) (2018) 1431. doi:10.3390/en11061431.

URL http://www .mdpi.com/1996-1073/11/6/1431

[23] T. Gafurov, M. Prodanovic, J. Usaola, PV system model reduction for reliability assessment studies, in: 2013 4th IEEE/PES Innovative Smart Grid Technologies Europe, ISGT Europe 2013, IEEE, Lyngby, Denmark, 2013, pp. 1-5. doi:10.1109/ISGTEurope.2013.6695420.

URL http://ieeexplore.ieee.org/document/6695420/ 
[24] L. Wang, W. Long, Dynamic model reduction of power electronic interfaced generators based on singular perturbation, Electric Power Systems Research 178 (2020) 106030. doi:10.1016/j.epsr.2019.106030.

URL https://linkinghub.elsevier.com/retrieve/pii/ S0378779619303499

[25] M. Rasheduzzaman, J. A. Mueller, J. W. Kimball, ReducedOrder Small-Signal Model of Microgrid Systems, IEEE Transactions on Sustainable Energy 6 (4) (2015) 1292-1305. doi:10.1109/TSTE.2015.2433177.

URL http://ieeexplore.ieee.org/document/7120179/

[26] M. Kudryavtsev, E. Rudnyi, J. Korvink, D. Hohlfeld, T. Bechtold, Computationally efficient and stable order reduction methods for a largescale model of MEMS piezoelectric energy harvester, Microelectronics Reliability 55 (5) (2015) 747-757. doi:10.1016/j.microrel.2015.02.003. URL https://linkinghub.elsevier.com/retrieve/pii/ S0026271415000232

[27] Z. Zhu, G. Geng, Q. Jiang, Power System Dynamic Model Reduction Based on Extended Krylov Subspace Method, IEEE Transactions on Power Systems 31 (6) (2016) 4483-4494. doi:10.1109/TPWRS.2015.2509481.

URL http://ieeexplore. ieee.org/document/7381702/

[28] G. Scarciotti, Low Computational Complexity Model Reduction of Power Systems With Preservation of Physical Characteristics, IEEE Transactions on Power Systems 32 (1) (2017) 743-752. doi:10.1109/TPWRS.2016.2556747.

URL https://ieeexplore.ieee.org/document/7466074/

[29] U. Baur, P. Benner, L. Feng, Model Order Reduction for Linear and Nonlinear Systems: A System-Theoretic Perspective, Archives of Computational Methods in Engineering 21 (4) (2014) 331-358. doi:10.1007/s11831-014-9111-2.

URL http://link.springer.com/10.1007/s11831-014-9111-2

[30] K. J. K. J. Aström, Feedback systems : an introduction for scientists and engineers, Princeton University Press, Princeton, N.J. ; Woodstock, 2008 . 
URL https://www.cds.caltech.edu/ murray/books/AM05/pdf/ am08-complete_22Feb09.pdf

[31] W. H. a. Schilders, H. a. V. D. Vorst, J. Rommes, Model Order Reduction: Theory, Research Aspects and Applications, 1st Edition, Vol. 13 of Mathematics in Industry, Springer Berlin Heidelberg, Berlin, Heidelberg, 2008. arXiv:arXiv:1011.1669v3, doi:10.1007/978-3-540-78841-6. URL http://link. springer.com/10.1007/978-3-540-78841-6

[32] K. Hou, H. Jia, X. Xu, Z. Liu, Y. Jiang, A Continuous Time Markov Chain Based Sequential Analytical Approach for Composite Power System Reliability Assessment, IEEE Transactions on Power Systems 31 (1) (2016) 738-748. doi:10.1109/TPWRS.2015.2392103. URL http://ieeexplore.ieee.org/document/7024191/

[33] T. Kaczorek, Positive 1D and 2D Systems, 1st Edition, Communications and Control Engineering, Springer London, London, 2002. doi:10.1007/978-1-4471-0221-2.

URL http://link.springer.com/10.1007/978-1-4471-0221-2

[34] J. Saak, M. Köhler, P. Benner, Matrix Equation Sparse Solver (2019). URL https://www.mpi-magdeburg.mpg.de/projects/mess

[35] K. Jbilou, A. Riquet, Projection methods for large Lyapunov matrix equations, Linear Algebra and its Applications 415 (2-3) (2006) 344358. doi:10.1016/j.laa.2004.11.004.

URL https://linkinghub.elsevier.com/retrieve/pii/ S0024379504004707

[36] M. Hached, K. Jbilou, Numerical solutions to large-scale differential Lyapunov matrix equations, Numerical Algorithms 79 (3) (2018) 741757. arXiv:1705.09362, doi:10.1007/s11075-017-0458-y.

URL http://link.springer.com/10.1007/s11075-017-0458-y

[37] V. Simoncini, A New Iterative Method for Solving Large-Scale Lyapunov Matrix Equations, SIAM Journal on Scientific Computing 29 (3) (2007) 1268-1288. doi:10.1137/06066120X.

URL http://epubs.siam.org/doi/10.1137/06066120X

[38] B. Retterath, S. Venkata, A. Chowdhury, Impact of time-varying failure rates on distribution reliability, International Journal of 
Electrical Power \& Energy Systems 27 (9-10) (2005) 682-688. doi:10.1016/j.ijepes.2005.08.011.

URL https://linkinghub.elsevier.com/retrieve/pii/ S0142061505000876

[39] Peng Wang, R. Billinton, Reliability cost/worth assessment of distribution systems incorporating time-varying weather conditions and restoration resources, IEEE Transactions on Power Delivery 17 (1) (2002) 260265. doi:10.1109/61.974216.

URL http://ieeexplore.iee.org/document/974216/

[40] J. Roychowdhury, Reduced-order modelling of linear time-varying systems, in: IEEE/ACM International Conference on Computer-Aided Design, Digest of Technical Papers, ACM Press, New York, New York, USA, 1998, pp. 92-95. doi:10.1145/288548.288581.

URL http://portal.acm.org/citation. cfm?doid=288548. 288581

[41] N. Lang, J. Saak, T. Stykel, Balanced truncation model reduction for linear time-varying systems, Mathematical and Computer Modelling of Dynamical Systems 22 (4) (2016) 267-281. doi:10.1080/13873954.2016.1198386.

URL http://www.tandfonline.com/doi/full/10.1080/13873954. 2016.1198386

[42] J. Anderson, Distance to the nearest stable Metzler matrix, in: 2017 IEEE 56th Annual Conference on Decision and Control (CDC), Vol. 2018-Janua, IEEE, Melbourne, VIC, Australia, 2017, pp. 6567-6572. doi:10.1109/CDC.2017.8264649.

URL http://ieeexplore.ieee.org/document/8264649/

[43] T. Kaczorek, Positive stable realizations with system Metzler matrices, Archives of Control Sciences 21 (2) (2011) 167-188. doi:10.2478/v10170010-0038-z.

URL http://journals.pan.pl/dlibra/publication/97602/ edition/84189/content

[44] University of Bath, Balena HPC cluster (2020).

URL https://www.bath.ac.uk/corporate-information/ balena-hpc-cluster/ 
[45] N. Hadjsaïd, J. C. Sabonnadière, Electrical Distribution Networks, John Wiley \& Sons, Inc., Hoboken, NJ, USA, 2013. doi:10.1002/9781118601280.

URL http://doi.wiley.com/10.1002/9781118601280

[46] R. Billinton, W. Li, Reliability Assessment of Electric Power Systems Using Monte Carlo Methods, Springer US, Boston, MA, 1994. doi:10.1007/978-1-4899-1346-3.

URL http://link. springer. com/10.1007/978-1-4899-1346-3

[47] A. B. Ocnasu, Y. Besanger, P. Carer, R. Edf, D. France, Distribution System Availability Assessment Monte Carlo and Antithetic Variates Method, 19 th International Conference on Electricity Distribution (0268) (2007) 21-24.

URL http://www.cired.net/publications/cired2007/search. html

[48] R. Billinton, P. Wang, Reliability-network-equivalent approach to distribution-system-reliability evaluation, IEE Proceedings: Generation, Transmission and Distribution 145 (2) (1998) 149-153. doi:10.1049/ip-gtd:19981828.

URL https://digital-library.theiet.org/content/journals/ 10.1049/ip-gtd\{\_\}19981828

[49] R. Billinton, S. Kumar, N. Chowdhury, K. Chu, K. Debnath, L. Goel, E. Khan, P. Kos, G. Nourbakhsh, J. Oteng-Adjei, A reliability test system for educational purposes - basic data, IEEE Transactions on Power Systems 4 (3) (1989) 1238-1244. doi:10.1109/59.32623.

URL http://ieeexplore. ieee.org/document/32623/

[50] M. Ndawula, S. Djokic, I. Hernando-Gil, Reliability Enhancement in Power Networks under Uncertainty from Distributed Energy Resources, Energies 12 (3) (2019) 531. doi:10.3390/en12030531.

URL http://www .mdpi .com/1996-1073/12/3/531

[51] I. Hernando-Gil, I. S. Ilie, S. Z. Djokic, Reliability planning of active distribution systems incorporating regulator requirements and networkreliability equivalents, IET Generation, Transmission and Distribution 10 (1) (2016) 93-106. doi:10.1049/iet-gtd.2015.0292. 